\title{
Realistic beamforming design using SRS-based channel estimate for ns-3 5G-LENA module
}

\author{
Biljana Bojović \\ Sandra Lagén \\ Lorenza Giupponi \\ \{bbojovic,slagen,lgiupponi\}@cttc.es \\ Centre Tecnològic de Telecomunicacions de Catalunya (CTTC/CERCA) \\ Castelldefels, Barcelona, Spain
}

\begin{abstract}
Beamforming (BF) is a key procedure to overcome propagation limits in millimeter-wave bands but also to extend network coverage in sub $6 \mathrm{GHz}$ bands, as considered in recent 3GPP and IEEE communication standards. Up to date, ns-3 included various ideal BF methods, in which an ideal channel state acquisition is assumed and no BF overhead is considered. However, ns-3 lacks the implementation of realistic BF methods, in which the overhead needed to perform BF-related procedures and the errors due to a non-ideal channel state acquisition are taken into account. A way to perform realistic $\mathrm{BF}$ is to rely on Sounding Reference Signals (SRS) for channel estimation and then determine the best BF vectors based on the channel estimate. In this paper, we first present an abstraction model to perform BF using SRS-based channel estimation, and then we provide the implementation details of $5 \mathrm{G}$ New Radio (NR)-compliant SRSs and new realistic BF methods using SRS-based channel estimate, as included in the ns-3 5G-LENA simulator. Simulations are provided to compare realistic $\mathrm{BF}$ and ideal BF methods, for different propagation scenario conditions and SRS transmitted powers.
\end{abstract}

\section{CCS CONCEPTS}

- Networks $\rightarrow$ Network simulations; Mobile networks. KEYWORDS

ns-3, NR, 3GPP, SRS, realistic beamforming, TDD.

ACM Reference Format:

Biljana Bojović, Sandra Lagén, and Lorenza Giupponi. 2021. Realistic beamforming design using SRS-based channel estimate for ns-3 5G-LENA module. In 2021 Workshop on ns-3 (WNS3 2021), June 21, 2021, Virtual Event. ACM, New York, NY, USA, 7 pages. https://doi.org/xxxxxx.xxxxxxxxxxxxxx

\section{INTRODUCTION}

The use of multiple antennas in communication systems (a.k.a. Multiple-Input Multiple-Output (MIMO) systems) has attracted a lot of attention in the recent decades. MIMO permits increasing the data rate by sending multiple data streams simultaneously (known

Permission to make digital or hard copies of all or part of this work for personal or classroom use is granted without fee provided that copies are not made or distributed for profit or commercial advantage and that copies bear this notice and the full citation on the first page. Copyrights for components of this work owned by others than the author(s) must be honored. Abstracting with credit is permitted. To copy otherwise, or republish, to post on servers or to redistribute to lists, requires prior specific permission and/or a fee. Request permissions from permissions@acm.org.

WNS3 2021, June 21, 2021, Virtual Event

(C) 2021 Copyright held by the owner/author(s). Publication rights licensed to ACM. ACM ISBN $x x x x x x x x x x x x x x x x x x x x x x \ldots \$ 15.00$

https://doi.org/Xxxxxx.Xxxxxxxxxxxxx as spatial multiplexing) or increasing the robustness of the data transmission by sending replicated data (known as transmit diversity) or providing array gain into specific spatial areas by properly designing the antenna weights or beamforming vectors (known as beamforming) [7, 17]. Beamforming (BF) is particularly essential at high carrier frequencies within the millimeter wave (mmWave) region to combat the high pathloss propagation losses and blocking effects $[6,12,20]$. In case of $\mathrm{BF}$, a single spatial stream is sent per receiver and the multiple antennas are used to concentrate the radiated power towards the receiver's location, thus improving the received Signal to Interference plus Noise Ratio (SINR) and the probability of error at the target receiver, as well as reducing the generated interference towards other spatial locations. BF has been adopted in many recent communication standards, like IEEE 802.11 (Wi-Fi), IEEE 802.16 (WiMAX), 3GPP Long Term Evolution (LTE) (4G), and 3GPP New Radio (NR) (5G). IEEE 802.11ad/ay defines many mandatory and optional BF-related procedures [9, 16, 22]. 3GPP LTE covers BF in the so-called Transmission Mode 7 [21]. 3GPP NR standard has defined multiple beam management procedures $[1,10]$, including beam measurement, beam sweeping, beam reporting, and beam determination, for both Time Division Duplexing (TDD) and Frequency Division Duplexing (FDD) systems, in sub 6 and mmWave bands.

3GPP NR has introduced Channel State Information Reference Signals (CSI RSs) and Synchronization Signal Blocks (SS blocks) for beam management in the Downlink (DL) and Sounding Reference Signals (SRSs) for beam management in the Uplink (UL) $[1,10]$. However, in TDD systems, SRS can be used for beam management in both DL and UL directions because, due to channel reciprocity, beam reciprocity can be assumed (i.e., BF vectors for UL and DL are the same). This way, the SS blocks can be limited to the initial access and the use of CSI RSs for channel estimation at the user (UE in 3GPP) can be avoided. In the TDD case, SRS receptions at the base station (gNB in 3GPP NR) can be used to estimate the channel and, based on the channel estimation, the $\mathrm{BF}$ vectors can be determined for DL and UL. In this paper, we focus on 3GPP NR and TDD systems.

The ns-3 mmWave module for 5G NR in mmWave bands [15], as well as the ns-3 5G-LENA module for 5G NR in sub 6 and mmWave bands $[18,19]$, included various ideal BF methods. Those methods are ideal in the sense that no resources are employed to determine the best beams and an ideal channel estimation is assumed. However, as of today, both modules lack the implementation of realistic $\mathrm{BF}$ methods to correctly assess the additional overhead needed 
to perform beam management procedures and analyze the errors induced by a non-ideal channel estimation.

In this paper, we propose, implement, and evaluate a realistic beam management procedure for system-level simulation of $5 \mathrm{G} \mathrm{NR}$ TDD systems in ns-3. First, we have implemented NR-compliant SRS signals transmissions and receptions in the ns-3 5G-LENA module. Based on SRS reception, we propose an abstraction model to estimate the channel matrix in a multi-antenna system. The abstraction model is used, as in typical network-level simulations, to avoid the related signal processing operations and reduce its computational complexity. Using the SRS-based channel estimation, the $\mathrm{BF}$ vectors can be determined at the gNB side. For that, we have linked the SRS reception with the beam management classes at the gNB, enabling the realistic BF procedure. Finally, we have evaluated the proposed procedure and compared it with ideal BF methods, currently available in ns-3 mmWave and 5G-LENA modules.

The rest of the paper is organized as follows. Section 2 reviews different BF methods, the SRS defined in 3GPP NR specification, and its antecedents in ns-3. Also, we present an abstraction model to determine the BF weights using SRS-based channel estimation. Then, in Section 3, we describe the implementation of the NRcompliant SRS and the implementation of the realistic BF using SRS-based channel estimation. Examples and tests are described in Section 4. Simulation results are shown in Section 5. Finally, conclusions are drawn in Section 6.

\section{SRS-BASED BEAMFORMING}

In this section we review BF methods and SRS design in 3GPP NR, the two key ingredients to implement a realistic BF algorithm using SRS-based channel estimate. We also describe the antecedents in ns-3. In brief, SRS are available in ns-3 LENA but used for link adaptation, not for beam management, while ideal BF methods are available in ns-3 mmWave and ns-3 5G-LENA modules, but both modules lack realistic BF models. After that, we present an abstraction model for channel estimation using SRS in TDD systems, which is the key part to link the two ingredients.

\subsection{Beamforming methods}

In general, there are three basic methods to find the BF weight vector:

- Design the BF vector using direction of arrival (DoA). There are methods that allow estimating the DoA at the gNB from a UE transmitted signal, and so determine the UE position. Such DoA can then be used to steer the beam towards the UE location.

- Design the BF vector using channel estimation. The optimal BF weight can be found from the channel matrix. The gNB can directly estimate the channel from an uplink signal transmission from the UE, and use such a channel estimation for nearly optimal BF design. Thanks to channel reciprocity in TDD systems, such BF vectors are valid for both DL and UL transmissions. In 3GPP LTE and NR, the SRS have been specifically designed to be used for that purpose.

- Design the BF vectors by using beam training and receiver's feedback. In case that the gNB has a set of pre-defined beams, it can send training signals through all of them, so that the
UE can measure the received signal quality and then report the index of the best beam. Such procedures are standardized in both IEEE 802.11ad/ay and 3GPP NR.

\subsection{Sounding Reference Signal}

SRS is a reference signal sent in the UL (from UE to gNB) in 3GPP NR to measure the channel quality. In particular, SRS receptions at the gNB provide information about the combined effect of multipath fading and power loss of the transmitted signal from the UE. Based on SRS reception, the gNB can take smart decisions for resource allocation and scheduling, link adaptation (e.g., modulation and coding scheme selection) [8], inter-cell interference management [11], and beam management [10].

According to 3GPP NR specification [3], SRS transmissions can be periodic, aperiodic, or semi-persistent. In time domain, the range for SRS transmission in a slot is from 8th to 13th OFDM symbol. NR leaves the 14th symbol in a slot for PUCCH. Within such a 6 OFDM symbol region, SRS transmissions can take maximum 4 OFDM symbols in time domain. In frequency domain, the bandwidth for SRS can be adjusted and interleaving is permitted. Interleaving is defined by the so-called transmission combination, which defines the frequency distance between two subcarriers used by SRS. Based on such pattern, multiple SRS signals (from different antenna ports or UEs) can be multiplexed in the same OFDM symbol.

\section{3 ns-3 antecedents}

The mmWave module [15] introduced various BF methods for mmWave bands in ns-3. Namely, the long-term covariance matrix method and the beam search method. In the long-term covariance matrix method, BF vectors are computed from the maximal eigenvectors of the channel covariance matrix. In the beam search method, it is assumed that there is a discrete number of beams from a pre-designed codebook, from which the beam that provides the largest SINR (considering the beam and the channel matrix) is selected for each gNB-UE link. Also, a line-of-sight (LOS) path method was included in the mmWave module, in which the DoA was assumed to be perfectly known and used to steer the BF vector towards such direction. In the 5G-LENA module [18] (and in its extension for unlicensed spectrum operation [19]), we have available the beam search method and the LOS path method. Also, we introduced the representation for a quasi-omnidirectional $\mathrm{BF}$, and multiple combinations with the previous methods (e.g., the gNB using beam search method and the UE using quasi-omni reception). However, all the methods available in ns-3 NR modules [15, 18, 19] up to date, were ideal BF methods in the sense that they assume perfect knowledge of the channel matrix and do not consume any time/frequency overhead to design the BF vectors.

SRS are implemented in ns-3 LENA module [8]. The implemented model uses periodic SRS. SRS are sent by the UE with a pre-configured periodicity (SrsPeriodicity attribute in LteEnbRrc that defaults to $40 \mathrm{~ms}$ ), spanning over the whole bandwidth (not interleaved) and sent over the last symbol of the subframe (14th OFDM symbol). The RRC model includes an algorithm to dynamically assign a periodicity as a function of the number of UEs attached to an eNB, which outputs the SRS subframe offset and the SRS periodicity for each UE. In ns-3 LENA module, SRS are used by 
the eNB for link adaptation, i.e., to determine the UL MCS, through the computation of an UL CQI that uses the SINR received during the SRS transmission.

Both mmWave and 5G-LENA modules were missing SRS implementation. In addition, in 5G-LENA our objective by including SRS is different as compared to that of LENA. We want to use SRS for beam management purposes, in addition to link adaptation. For beam management, we can use the SRS to estimate the channel in multi-antenna TDD systems, assuming channel reciprocity (DL channel matrix equals to the transpose of the UL channel matrix) and use the channel estimate for BF design. For that, we cannot rely on the received signal from an SRS transmission, since this is not modeled in ns-3 spectrum. Instead, we need an abstraction model for SRS-based channel estimation.

\subsection{Abstraction model for SRS-based channel estimation}

Assume a single-antenna system. Let $h$ denote the (complex-valued) small-scale fading channel between a UE and a gNB. Then, the estimation of the small-scale fading channel at the gNB can be modeled as [14]:

$$
\hat{h}=\alpha(h+e)
$$

where $\alpha$ is a scaling factor to maintain normalization of the estimated channel, and $e$ is the white complex Gaussian channel estimation error.

The estimation error $(e)$ is assumed to be characterized by zeromean and variance $\sigma_{e}^{2}$. The variance of the error is given by

$$
\sigma_{e}^{2}=\frac{1}{(\operatorname{SINR}+\Delta)}
$$

where SINR is the received SINR of SRS at the gNB and $\Delta$ is the gain obtained from time-domain filtering during the channel estimation. According to $3 \mathrm{GPP}$ analysis of SRS transmission, $\triangle$ is set to $9 \mathrm{~dB}$ [14]

The scaling factor $(\alpha)$ is given by

$$
\alpha=\sqrt{\frac{1}{\left(1+\sigma_{e}^{2}\right)}} .
$$

In case of multi-antenna systems, assuming independent smallscale fading among antenna elements, the model in (1) can be used to estimate each fading component of the 3GPP spatial channel matrix [23]. Then, the channel matrix estimate can be used to compute transmit/receive BF vectors, as part of the beam management.

\section{IMPLEMENTATION IN NS-3}

In this section we provide the implementation details of SRS allocation, transmission, and reception, as implemented in ns-3 5G-LENA simulator. Also, we describe the developed extension of the beamforming framework and the implementation of the new realistic $\mathrm{BF}$ procedure, which uses the abstraction model presented in Section 2.4 to estimate the channel based on SRS report.

\subsection{SRS transmission and reception}

SRS transmission typically spans over 1, 2 or 4 consecutive OFDM symbols at the end of the NR slot. 5G-LENA implements such behaviour in the time domain by allowing different configurations. In the frequency domain, in order to allow frequency multiplexing,

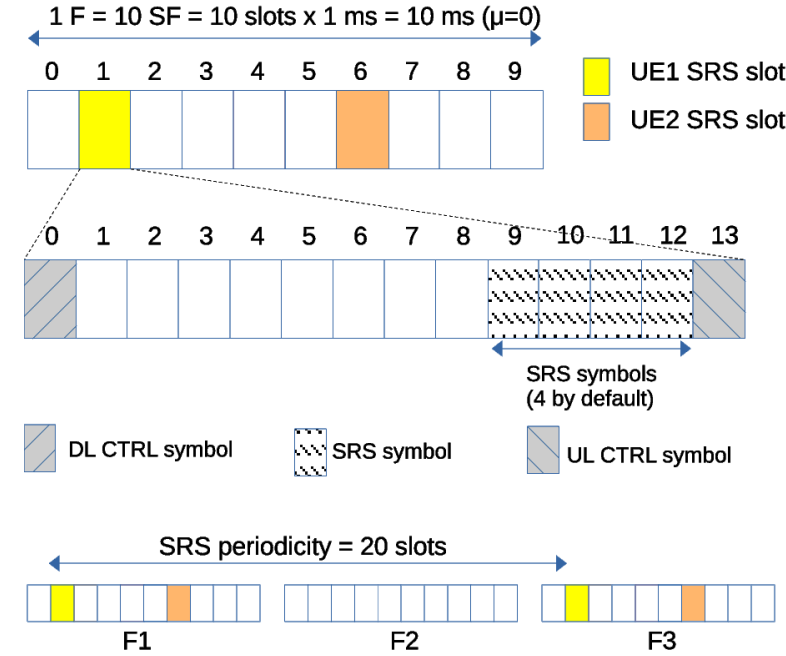

Figure 1: Example of SRS transmissions of 4 different UEs (maximum 1 UE SRS transmission per slot, as per 5G-LENA design), considering SRS periodicity equal to 20 slots. Numerology considered is $\mu=0$. F stands for frame and SF for subframe.

SRS is typically transmitted over only a subset of subcarriers, defined by the configuration, e.g., each 2 nd or each 4 th subcarrier is used for SRS transmission. However, since the minimum transmission granularity in 5G-LENA module is a RB in frequency domain, all subcarriers are used for SRS transmission. Figure 1 shows the slot structure and the symbols over which the SRS transmission spans, assuming a repeated TDD pattern structure of [DL F UL UL UL] (i.e., one DL slot, followed by one flexible slot and three UL slots $^{1}$ ) and that SRS transmissions occur in F slots (i.e., slots number 1 and 6 in the figure).

In $5 \mathrm{G} \mathrm{NR}$, SRS parameters, such as periodicity and offset, are typically configured by RRC and notified to UE [2]. Another option is to have gNB MAC scheduler to determine the SRS periodicity/offset and then to notify UE through DCI format 2_3 on which resources SRS should be transmitted [4]. The latter option, scheduling-based SRS, is a more dynamic approach and allows more flexible SRS parameter and periodicity assignment, e.g., when there are less UEs, a lower periodicity value can be used, while when there are more UEs, the gNB MAC scheduler can dynamically increase the periodicity and then update the offsets accordingly. We have implemented scheduling-based SRS.

To allow dynamic SRS scheduling and adjustment of SRS periodicity/offset of all UEs, we introduced NrMacSchedulerSrs and NrMacSchedulerSrsDefault into 5G-LENA model. NrMacSchedulerSrs is an interface that is used by the NR gNB MAC scheduler to obtain the SRS offset/periodicity for a UE. There can be various implementations of this interface that would simulate different algorithms for SRS offset/periodicity generation. In NrMacSchedulerSrsDefault, we provide one of possible implementations. Each

\footnotetext{
${ }^{1}$ In 5G-LENA, flexible slots consist of DL/UL control symbols and a variable number of DL and UL symbols for data; DL slots carry only DL control and DL data; and UL slots consist of UL data and UL control parts.
} 
time a new UE is attached it is called the function AddUe that returns the offset/periodicity configuration. When scheduler detects that the SRS periodicity is too small for the number of UEs it calls function IncreasePeriodicity, which picks up the next periodicity value from the list of standard values, i.e., 2, 4, 5, 8, 10, 16, 20, 32, $40,64,80,160,320,640,1280,2560$ slots [2]. Scheduling-based SRS is more flexible approach than SRS configuration through RRC. E.g., in 4G-LENA SRS configuration is through RRC and static, which requires that a user needs to configure SRS periodicity based on the maximum expected number of UEs in the simulation scenario. To allow dynamic SRS periodicity adaptation in 5G-LENA, it was necessary to set a constraint which is that at most $1 \mathrm{UE}$ can send the SRS in a single slot.

Configuration parameters related to SRS transmissions are specified in NrMacSchedulerNs3 class. The user can configure the number of SRS symbols that will be allocated for SRS transmission through the attribute SrsSymbols. Additionally, the user can configure whether SRS will be transmitted only in flexible slots, or in both flexible and UL slots by setting the attribute EnableSrsInUISlots.

\subsection{Realistic beamforming}

5G-LENA BF framework is extended to provide support for realistic $\mathrm{BF}$ algorithms. While ideal $\mathrm{BF}$ algorithms decide the beams based on either the assumptions of perfect knowledge of the channel (e.g., cell scan method) or the exact positions of the devices (e.g., DoA method), the realistic $\mathrm{BF}$ algorithms are expected to select the best beam based on some measurements, and this is the main difference between these two types of algorithm implementations in 5G-LENA. Various ideal BF algorithms can be implemented by inheriting IdealBeamformingAlgori thm class. To implement a new realistic BF algorithm, we have created a separate class called RealisticBeamformingAlgorithm. Both classes, implement BeamformingAlgorithm interface and thus both must have the function GetBeamformingVectors which determines BF vectors to be used on a pair of devices, i.e., gNB and UE. For example, CellScanBeamforming implements a type of ideal BF algorithm that searches for the best pair of BF vectors from the set of pre-defined BF vectors assuming the perfect knowledge of the channel. On the other hand, RealisticBeamformingAlgori thm uses SRS SINR measurements. Similarly to previously mentioned CellScanBeamforming there is a set of pre-defined BF vectors, but the knowledge of the channel is not perfect, and depends on the quality of reported SRS measurement. Another difference between ideal BF algorithm implementations and the realistic one, is that ideal BF vectors of all devices are updated at the same time based on the configured periodicity through BeamformingPeriodicity attribute of IdealBeamformingHelper. On the other hand, when RealisticBeamformingAlgorithm is used, the $\mathrm{BF}$ vectors are updated when the configured trigger event occurs, and only the BF vectors of the pair of devices for which SRS measurement has been reported (pair of $\mathrm{gNB}$ and UE) are updated. The BF vector trigger update event can be either SRS count event (e.g., after every N SRSs are received, the BF vectors are updated), or based on the delay event after SRS reception (e.g., $\delta$ time after each SRS reception). The type of event and its parameters can be configured through RealisticBfManager class. Hence, in order to use realistic BF functionality it is necessary to install RealisticBfManager at gNBs PHY instead of the default BeamManager class. The configuration of trigger event and its parameters can be done per gNB instance granularity, but can be easily extended to be done per UE.

When UE is attached to gNB, a BF task is created. The BF task is composed of a pair of connected devices for which the realistic $\mathrm{BF}$ helper will manage the update of the BF vectors by calling GetBeamformingVectors of configured realistic BF algorithm. Also, for each task is created an instance of realistic BF algorithm, which is then connected to NrSpectrumPhy SRS SINR trace to receive $\mathrm{SRS}$ reports. Realistic BF algorithm is also connected to its helper through callback to notify it when BF vectors of device pair needs to be updated (based on configuration and SRS reports). When $\mathrm{BF}$ vectors need to be updated, the function GetBeamformingVector or realistic BF algorithm is called, which calls GetEstimatedLongTermComponent for each pair of pre-defined beams of the receiver and transmitter in order to estimate the channel quality of each of them based on the SRS reports. The estimation of the channel is done based on the abstraction model explained in Section 2.4. Finally, CalculateTheEstimatedLongTermMetric calculates the metric that is used to select the best BF pair.

In Figure 2, we show the diagram of the classes that are used for realistic BF based on SRS measurements, the dependencies among classes, and the most important methods. E.g., we can see that RealisticBeamformingAlgorithm needs to access to ThreeGppChannelModel to obtain the channel matrix in order to perform the estimation of the channel based on SRS report.

\section{EXAMPLES AND TESTS}

The example cttc-realistic-beamforming.cc included in the $\mathrm{nr}$ module demonstrates the usage of the proposed framework. It is a simulation script for the realistic BF evaluation. The topology is very simple: it consists of a single gNB and single UE, placed at a certain distance from each other and communicating over a wireless channel. Simulation allows to configure various parameters out of which the most important are: the distance (by configuring deltaX and deltaY parameters, which basically determine the position of the UE), the type of the BF method (ideal or real), the random run number (which will allow us to run many simulations and to average the results), the UE power, 3GPP scenario (Urban Macro, Urban Micro, Indoor Hotspot, etc). The output is saved in database (simulation configuration and average SINR). The database is created in the root project directory if not configured differently.

The test $\mathrm{nr}$-realistic-beamforming-test. cc included in the $\mathrm{nr}$ module tests the realistic BF implementation. It involves two devices, a transmitter and a receiver, placed at a certain distance from each other and communicating over a wireless channel at 28 $\mathrm{GHz}$ carrier frequency. The test compares the performance of two different BF methods: 1) the proposed realistic BF algorithm, which uses SRS reception to estimate the channel and computes $\mathrm{BF}$ weights based on such channel estimate, and 2) an ideal BF algorithm, which selects the BF weights assuming perfect knowledge of the channel matrix coefficients. The test checks that with low SINR from SRS, realistic $\mathrm{BF}$ algorithm makes more mistakes in channel estimation 


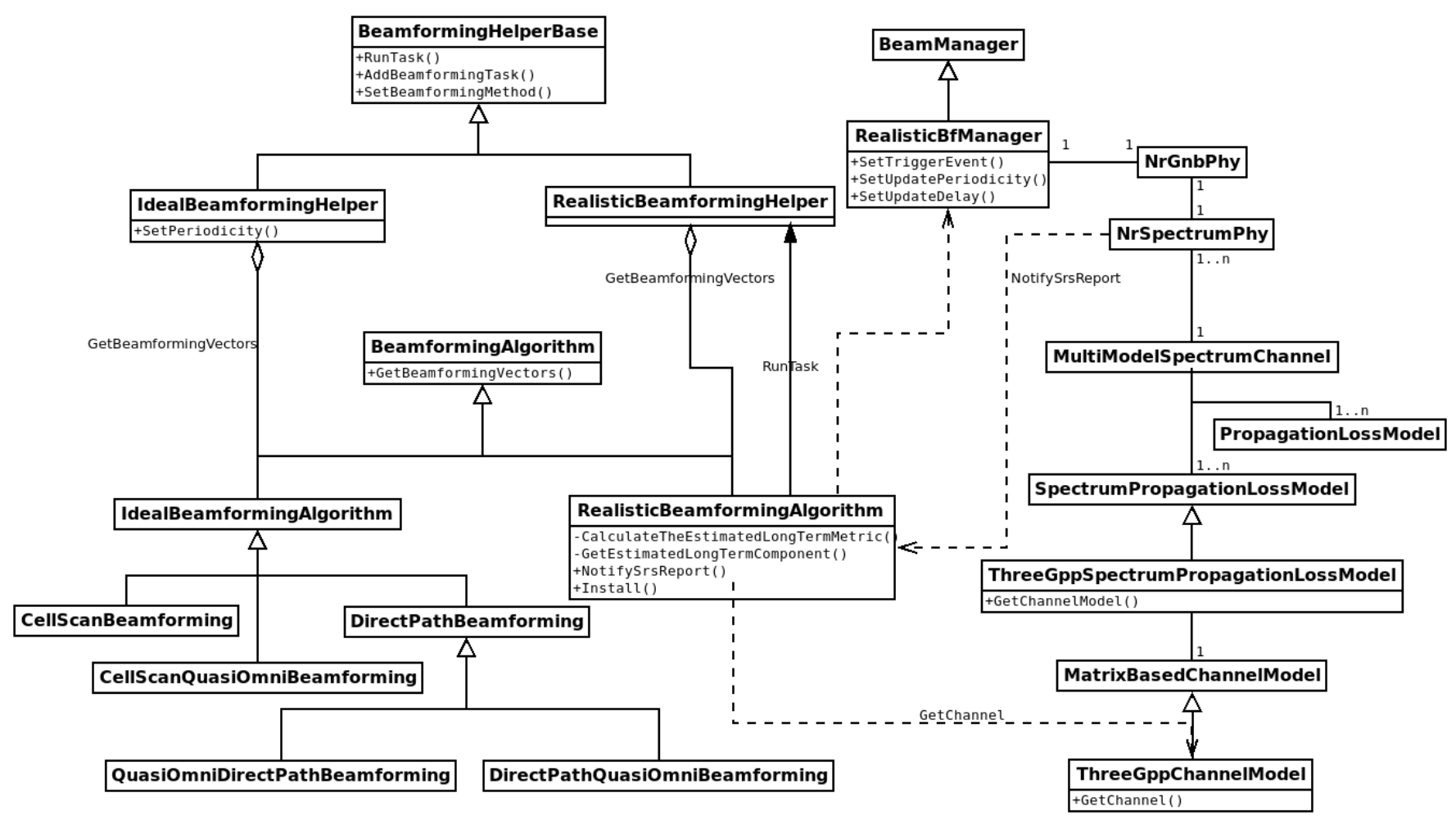

Figure 2: Diagram of realistic/ideal BF, dependencies on 3GPP channel related classes, and NrGnbPhy.

than ideal BF algorithm. Also, the test checks that with high SINR from SRS, realistic BF algorithm generates almost always the same decision as that of the ideal BF method, and so, the same pair of beams are selected for the two communicating devices.

\section{SIMULATION RESULTS}

For the performance assessment, we use ns-3 5G-LENA [18] with the 3GPP spatial channel model developed in [23], compliant with TR 38.901, and the NR-based PHY abstraction model in [13]. The deployment includes two devices, a gNB and a UE, placed at a certain distance from each other. We use three propagation scenarios: Urban Macro (UMa), Urban Micro (UMi), and Indoor Hostpot (InH), which affect the channel and propagation models, as per TR 38.901 [5]. Also, UMa is characterized by a gNB antenna height of $25 \mathrm{~m}$, in UMi the gNB antenna height is of $10 \mathrm{~m}$, and $\mathrm{InH}$ has a gNB antenna height of $3 \mathrm{~m}$. In UMa and UMi scenarios, we used a gNB transmit power of $35 \mathrm{dBm}$, while for $\mathrm{InH}$ scenario the gNB transmit power is set to $24 \mathrm{dBm}$. The UE has an antenna height of $1.5 \mathrm{~m}$. The transmission is performed in the $28 \mathrm{GHz}$ band region, using numerology 2 (i.e., $60 \mathrm{KHz}$ subcarrier spacing), with $100 \mathrm{MHz}$ channel bandwidth and a PRB overhead of 0.04 (typical of NR). The antenna array configuration consists of $4 \times 8$ directional antenna elements at the gNB and $2 \times 4$ directional antenna elements at the UE. Single MIMO layer is assumed. The duplexing mode is TDD, with dynamic downlink-uplink slot structure. The SRS are configured to use 1 OFDM symbol.
The simulations are performed by varying the 3D distance among the gNB and the UE. For UMa and UMi scenarios, we test up to $1 \mathrm{Km}$ distances, and for $\mathrm{InH}$ scenario, distances up to $200 \mathrm{~m}$ are considered. We compare ideal BF method using cell scan and the proposed realistic BF method using SRS-based channel estimate. Also, we test two different UE transmit powers: $0 \mathrm{dBm}, 10 \mathrm{dBm}$, and $20 \mathrm{dBm}$, to assess the impact of the UE power on the SRS report, and so on the beam selection. For each configuration of 3D distance and UE transmit power, we compute the average SINR received at the UE, in DL, to see the impact of the BF vector selection. Simulation results are averaged over 40 random channel realizations.

Fig. 3, Fig. 4, and Fig. 5 show the DL SINR as a function of the 3D gNB-UE distance for UMa, UMi, and InH scenarios, respectively. We have separated the Line-Of-Sight (LOS) channel condition ((a) subfigures) and the Non-Line-Of-Sight (NLOS) channel condition ((b) subfigures), to determine under which conditions the non-ideal channel estimation impacts the most.

Results show that with LOS conditions, in any of the considered scenarios (UMa, UMi, and InH), there is not an impact on the DL SINR when using realistic BF and a variety of UE transmit powers and gNB-UE distances (see Fig. 3.(a), Fig. 4.(a), and Fig. 5.(a)), meaning that the channel can be properly estimated and the same $\mathrm{BF}$ vectors are selected with both ideal and realistic BF methods. This is due to the good propagation channel under LOS conditions, which allow a good estimate of the channel matrices based on SRS measurements and, therefore, a proper BF vector selection. 

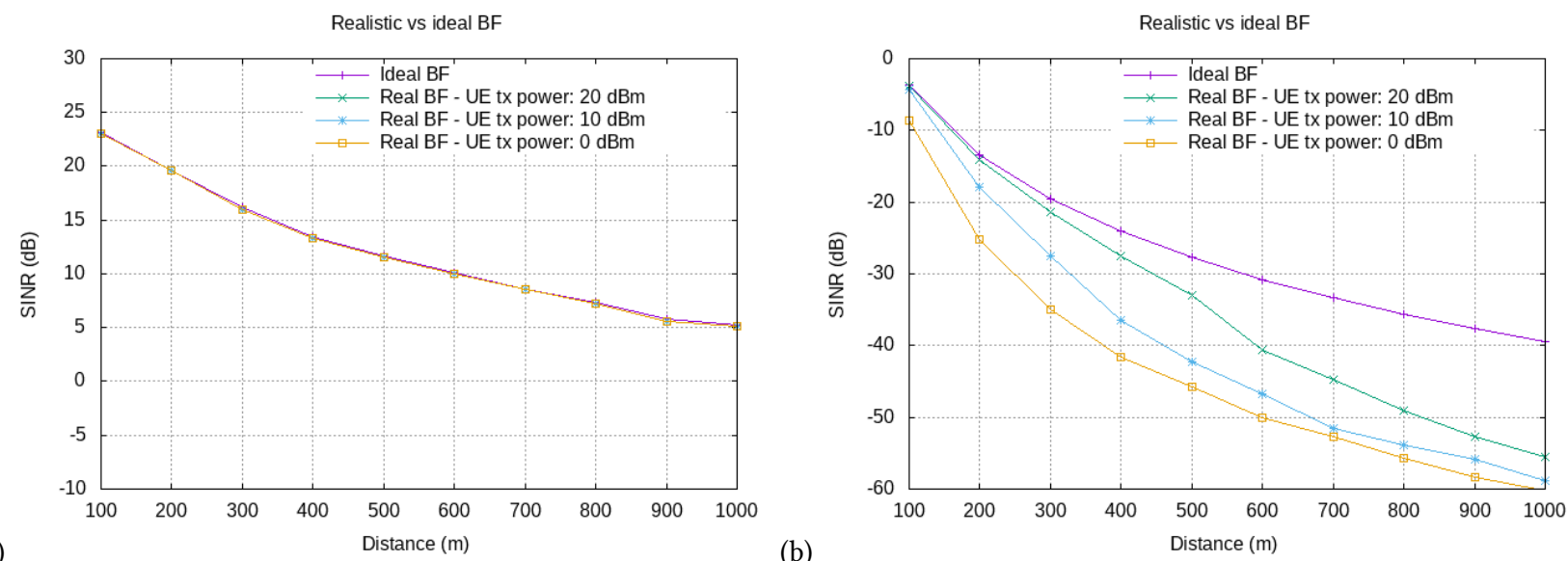

Figure 3: DL SINR (in dB) vs 3D distance (in meters). UMa scenario. (a) LOS, (b) NLOS.
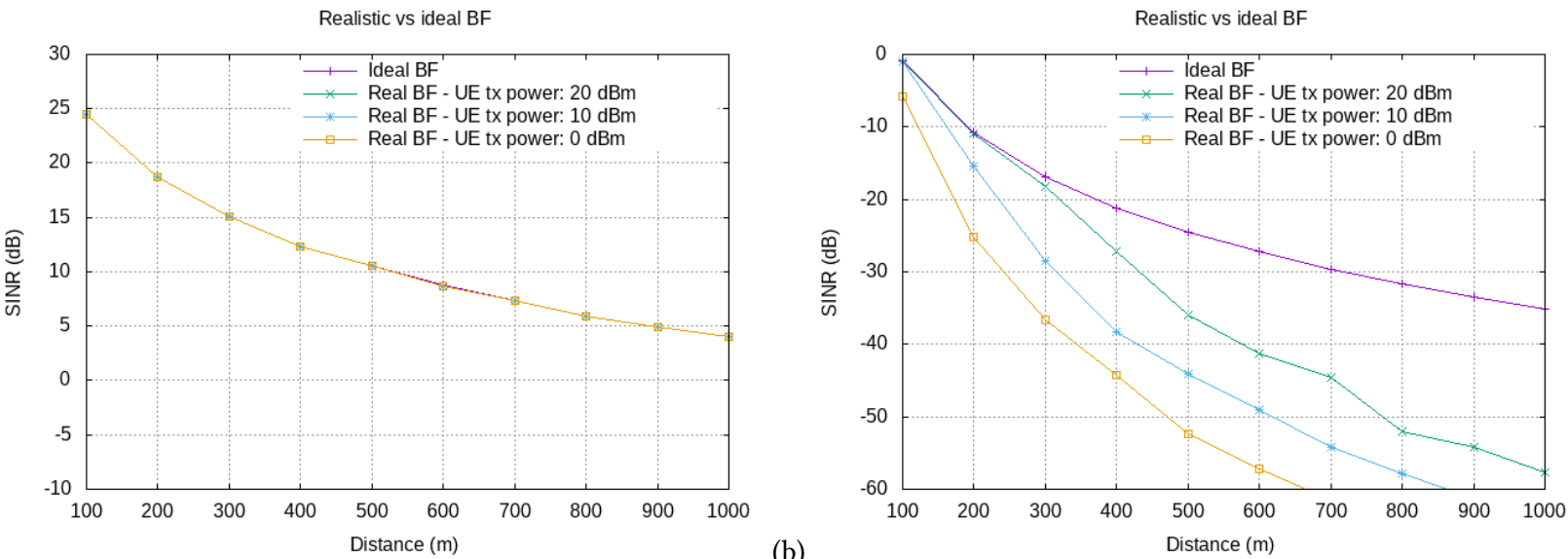

(a)

(b)

Figure 4: DL SINR (in dB) vs 3D distance (in meters). UMi scenario. (a) LOS, (b) NLOS.
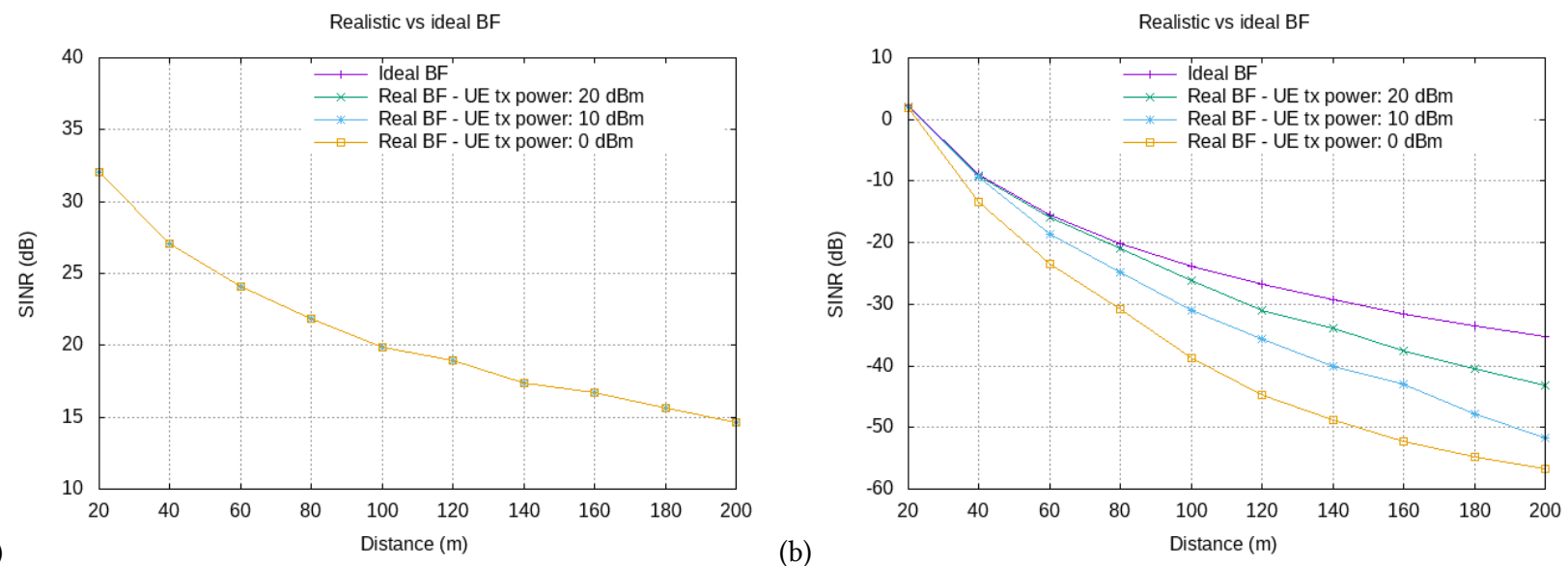

Figure 5: DL SINR (in dB) vs 3D distance (in meters). InH scenario. (a) LOS, (b) NLOS. 
However, in NLOS conditions, in all of the considered scenarios (UMa, UMi, and InH), we can see a degradation on the DL SINR due to the use of realistic BF (see Fig. 3.(b), Fig. 4.(b), and Fig. 5.(b)). The DL SINR loss is more evident as the 3D distance increases, because the SRS SINR report is also reduced with the distance, and so it negatively affects the BF selection. Such a DL SINR degradation is further increased in the case of using a lower UE transmit power for SRS transmissions (e.g., $0 \mathrm{dBm}$ ), as expected. This is because, at low SRS transmit power, the SRS SINR report value is reduced, and so the channel estimation has more errors and the BF vector selection differs more from the optimal one, which ultimately impacts negatively on the DL SINR. With a lower UE transmit power, the DL SINR degradation appears at shorter distances, as compared to scenarios in which the UE uses a higher UL transmit power. Thus, with high UE transmit powers, the real BF procedure can maintain the performance of the ideal BF over a wider range of distances. In fact, both in UMi NLOS and UMa NLOS scenarios, UE transmit powers of $20 \mathrm{dBm}$ (and higher) do not exhibit performance degradation up to $200 \mathrm{~m}$ distance. With $10 \mathrm{dBm}$ UE transmit power, the DL SINR degradation starts after $100 \mathrm{~m}$ distance in NLOS. Under InH NLOS scenario, transmit powers of $20 \mathrm{dBm}$ (and higher) do not exhibit performance degradation up to $60 \mathrm{~m}$ distance, while with $10 \mathrm{dBm}$ and $0 \mathrm{dBm}$ UE transmit powers, the DL SINR degradation starts after $40 \mathrm{~m}$ and $20 \mathrm{~m}$, respectively.

\section{CONCLUSIONS}

In this paper, we presented the extension of 5G-LENA module to support simulations with realistic BF procedures in TDD systems. First, we have presented an abstraction model to perform BF vector selection using SRS-based channel estimates. Then, we have provided the implementation details of the two key ingredients of realistic BF: SRS and realistic BF algorithms. Dynamic SRS scheduling and NR-compliant SRS transmission and reception have been implemented and detailed. On the other hand, the BF framework in 5G-LENA has been extended to account for realistic BF techniques. Finally, we have assessed the performance of realistic BF against ideal BF under Urban Macro, Urban Micro, and Indoor Hotspot propagation conditions, for various distances and SRS transmit powers. Simulation results have demonstrated the effectiveness of realistic BF procedures that use SRS measurements to estimate the channel matrices and perform BF vector selection. Also, the results have highlighted the conditions under which the real $\mathrm{BF}$ does not exhibit a significant BF vector selection difference, as compared to ideal BF. These conditions are LOS propagation conditions, and short distances and/or high UE transmit powers under NLOS propagation conditions.

\section{ACKNOWLEDGMENTS}

This work has been partially funded by Spanish MINECO grant TEC2017-88373-R (5G-REFINE) and Generalitat de Catalunya grant 2017 SGR 1195. The work received funding from the USA Defense Spectrum Organization (DSO) under the Spectrum Sharing Test and Demonstration (SSTD) Program in the context of the S3 project. The authors would also like to acknowledge Peter Barnes at Lawrence Livermore National Lab. and the S3 team for feedbacks and fruitful collaborations.

\section{REFERENCES}

[1] 3GPP. 2018. NR and NG-RAN Overall Description. TS 38.300 (Rel. 15). (2018).

[2] 3GPP. 2018. Radio Resource Control (RRC). TS 38.331 (Rel. 15). (2018).

[3] 3GPP. 2020. TSG RAN; NR; Physical channels and modulation. TS 38.211 (Rel. 15). (2020).

[4] 3GPP. 2021. TSG RAN; NR; Multiplexing and channel coding. TS 38.212 (Rel. 15). (2021).

[5] 3GPP. Jun. 2019. Study on Channel Model for Frequencies from 0.5 to $100 \mathrm{GHz}$. TR 38.901 (Rel. 15), V15.0.0. (Jun. 2019)

[6] J. G. Andrews, T. Bai, M. N. Kulkarni, A. Alkhateeb, A. K. Gupta, and R. W. Heath. 2017. Modeling and Analyzing Millimeter Wave Cellular Systems. IEEE Transactions on Communications 65, 1 (Jan 2017), pp. 403-430. https://doi.org/10. 1109/TCOMM.2016.2618794

[7] J. Bach Andersen. 2000. Antenna arrays in mobile communications: gain, diversity, and channel capacity. IEEE Antennas and Propagation Magazine 42, 2 (2000), pp. 12-16. https://doi.org/10.1109/74.842121

[8] Nicola Baldo, Marco Miozzo, Manuel Requena-Esteso, and Jaume Nin-Guerrero. 2011. An Open Source Product-oriented LTE Network Simulator Based on Ns-3. In Proceedings of the 14th ACM International Conference on Modeling, Analysis and Simulation of Wireless and Mobile Systems (MSWiM '11). ACM, New York, NY, USA, pp. 293-298.

[9] Y. Ghasempour, C. R. C. M. da Silva, C. Cordeiro, and E. W. Knightly. 2017. IEEE 802.11ay: Next-Generation $60 \mathrm{GHz}$ Communication for $100 \mathrm{~Gb} / \mathrm{s} \mathrm{Wi}$-Fi. IEEE Communications Magazine 55, 12 (Dec 2017), pp. 186-192. https://doi.org/10. 1109/MCOM.2017.1700393

[10] M. Giordani, M. Polese, A. Roy, D. Castor, and M. Zorzi. 2019. A Tutorial on Beam Management for 3GPP NR at mmWave Frequencies. IEEE Communications Surveys Tutorials 21, 1 (2019), pp. 173-196. https://doi.org/10.1109/COMST.2018. 2869411

[11] S. Lagen, A. Agustin, and J. Vidal. 2014. Decentralized Beamforming with Coordinated Sounding for Inter-Cell Interference Management. In European Wireless 2014; 20th European Wireless Conference. pp. 1-6.

[12] S. Lagen, L. Giupponi, S. Goyal, N. Patriciello, B. Bojovic, A. Demir, and M. Beluri. 2019. New Radio Beam-based Access to Unlicensed Spectrum: Design Challenges and Solutions. IEEE Communications Surveys Tutorials (2019), pp. 1-1.

[13] S. Lagen, K. Wanuga, H. Elkotby, S. Goyal, N. Patriciello, and L. Giupponi. 2020. New Radio Physical Layer Abstraction for System-Level Simulations of 5G Networks. In IEEE International Conference on Communications.

[14] F.-L. Luo and C. J. Zhang. 2016. Signal Processing for 5G: Algorithms and Implementations. John Wiley \& Sons. (Aug. 2016).

[15] M. Mezzavilla, M. Zhang, M. Polese, R. Ford, S. Dutta, S. Rangan, and M. Zorzi. 2018. End-to-End Simulation of 5G mmWave Networks. IEEE Communications Surveys \& Tutorials 20, 3 (Third quarter 2018), pp. 2237-2263.

[16] T. Nitsche, C. Cordeiro, A. B. Flores, E. W. Knightly, E. Perahia, and J. C. Widmer. 2014. IEEE 802.11ad: directional $60 \mathrm{GHz}$ communication for multi-Gigabit-persecond Wi-Fi [Invited Paper]. IEEE Communications Magazine 52, 12 (December 2014), pp. 132-141. https://doi.org/10.1109/MCOM.2014.6979964

[17] D. P. Palomar. 2003. A unified framework for communications through MIMO channels. Ph.D. Dissertation. Universitat Politècnica de Catalunya. PhD thesis.

[18] N. Patriciello, S. Lagen, B. Bojovic, and L. Giupponi. 2019. An E2E Simulator for 5G NR Networks. Simulation Modelling Practice and Theory 96 (2019), pp. 101933.

[19] N. Patriciello, S. Lagen, B. Bojović, and L. Giupponi. 2020. NR-U and IEEE 802.11 Technologies Coexistence in Unlicensed mmWave Spectrum: Models and Evaluation. IEEE Access 8 (2020), pp. 71254-71271. https://doi.org/10.1109/ ACCESS.2020.2987467

[20] Z. Pi and F. Khan. 2011. An introduction to millimeter-wave mobile broadband systems. IEEE Communications Magazine 49, 6 (June 2011), pp. 101--107.

[21] Rohde and Schwarz. [n. d.]. LTE Transmission Modes and Beamforming, White Paper. ([n. d.]).

[22] P. Zhou, K. Cheng, X. Han, X. Fang, Y. Fang, R. He, Y. Long, and Y. Liu. 2018. IEEE 802.11ay-Based mmWave WLANs: Design Challenges and Solutions. IEEE Communications Surveys Tutorials 20, 3 (thirdquarter 2018), pp. 1654-1681. https: //doi.org/10.1109/COMST.2018.2816920

[23] T. Zugno, M. Polese, N. Patriciello, B. Bojovic, S. Lagen, and M. Zorzi. 2020. Implementation of a spatial channel model for ns-3. ns-3 Workshop 2020 (June 2020). 